UCRL-JC-123074

PREPRINT

CONF-960155--6

\title{
Spectroscopic Temperature Measurements of Non-Equilibrium Plasmas
}

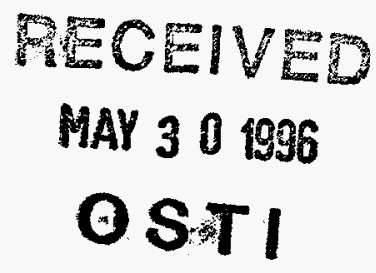

C. A. Back, S. H. Glenzer, R. W. Lee,

B. J. MacGowan, J. C. Moreno, J. K. Nash,

L. V. Powers, and T. D. Shepard

This paper was prepared for submittal to the

10th APS Topical Conference on Atomic Processes in Plasmas

San Francisco, CA

January 14-18, 1996

April 24, 1996

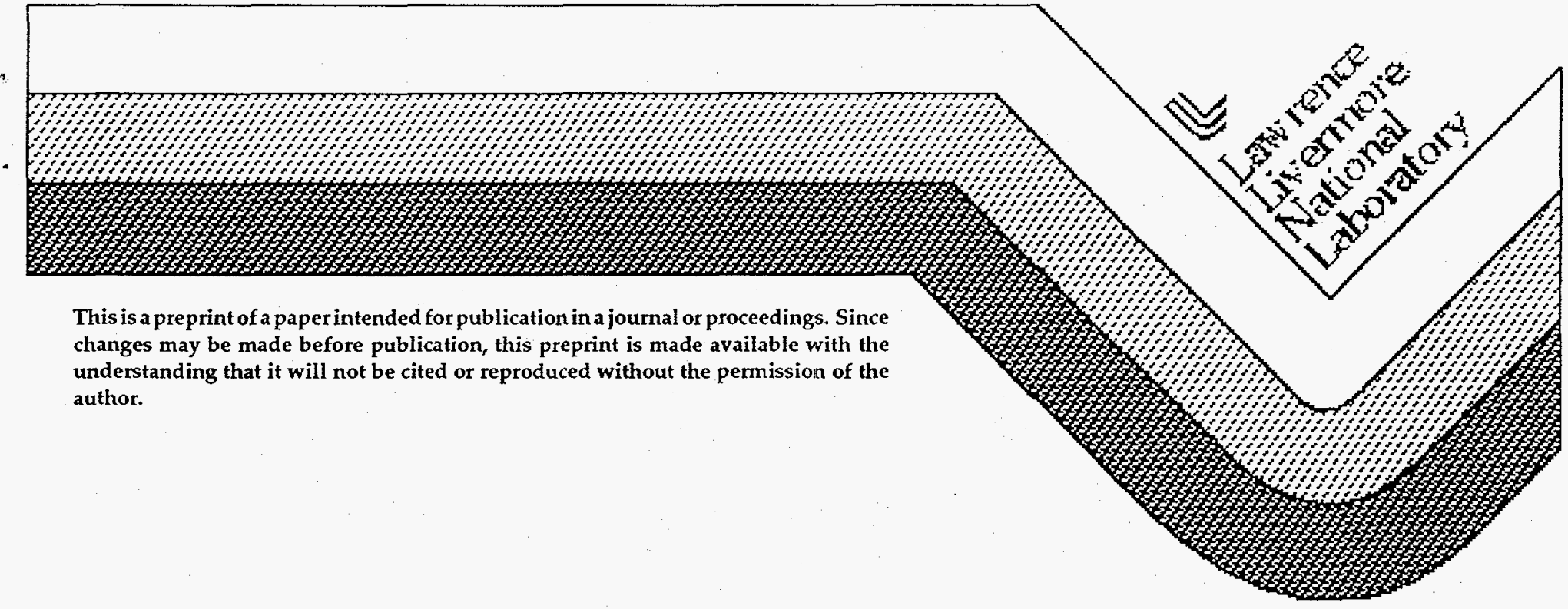




\section{DISCI.AIMER}

This document was prepared as an account of work sponsored by an agency of the Linited States Covernment. Neither the I nited States Government nor the Ciniversity of California nor any of their employees, makes any warranty, express or implied, or assumes any legal liability or responsibility for the accuracy, completeness, or usefulness of any information, apparatus, product, or process disclosed, or represents that its use would not infringe privately owned rights. Reference herein to any specific commercial products, process, or service by trade name. trademark, manufacturer, or otherwise, does not necessarily constitute or imply its endorsement, recommendation, or favoring by the linited States Government or the l'niversity of (alifornia. The views and opinions of authors expressed herein do not necessarily state or reflect those of the I'nited States Govermment thereof, and shall not he used for advertising or product endorsement purposes. 


\section{DISCLAIMER}

Portions of this document may be illegible in electronic image products. Images are produced from the best available original document. 


\title{
Spectroscopic Temperature Measurements of Non-Equilibrium Plasmas
}

\author{
C. A. Back, S. H. Glenzer, R. W. Lee, B. J. MacGowan, \\ J. C. Moreno, J. K. Nash, L. V. Powers, and T. D. Shepard
}

Lawrence Livermore National Laboratory, P.O. Box 808, Livermore, CA 94551

\begin{abstract}
The characterization of laser-produced plasmas has required the application of spectroscopic techniques to non-standard conditions where kinetics models have not been extensively tested. The plasmas are produced by the Nova laser for the study of inertial confinement fusion, can be $\mathrm{mm}$ in size, and evolve on sub-nanosecond time scales. These targets typically achieve electron temperatures from $2-4 \mathrm{keV}$ and electron densities of $10^{20}-10^{22} \mathrm{~cm}^{-3}$. We have measured the electron temperature of two types of targets: bags of gas and hohlraums, Au cylinders with laser entrance holes in the flat ends. By comparing data from different targets, we examine the time-dependence of spectroscopic plasma diagnostics.
\end{abstract}

Spectroscopy has evolved as an important diagnostic of laser-produced plasmas. Discrete transitions allows identification of different ionic species formed in the plasma by the wavelength of their emission. As the description of plasma constituents and their atomic details have become better understood, emission and absorption spectroscopy have been used to measure electron temperature, ionic temperature, electron density, and ionic populations (1).

In inertial confinement fusion research, lasers can produce extreme plasma conditions such as the high temperatures and densities found in stars. Since the hydrodynamic evolution of the plasmas takes place in extremely short time scales, diagnostics must be applied to difficult, non-steady state conditions. Furthermore, radiation fluxes of laboratory plasmas produce a regime in which collisionalradiative plasma models have not been well-tested. In such plasmas, the spectroscopic techniques must be carefully applied.

In these experiments, spectroscopy is used to diagnose the electron temperature, $\mathrm{T}_{e}$, of $\mathrm{mm}$-scale plasmas. Measurements of $\mathrm{T}_{e}$ enable a check of energy absorption, electron conduction, and overall energy balance in the hydrodynamic simulations. For example, parametric plasma processes are dependent on $T_{e}$ and scattering of laser light can lead to a significant loss of energy over the mm-long laser path lengths (2). Hence, measurements of $T_{e}$ give a direct measure of our success in modeling the hohlraum'environment (3).

Time-dependent analysis of laser-produced plasmas can be important because the plasmas are created by ns-long pulses. These plasmas ionize rapidly and can easily achieve $T_{e}>1 \mathrm{keV}$. However, the heating can create plasmas having 
densities too low to assure that a steady state model of the plasma kinetics is sufficient. The densities of the plasmas in this study can be varied by changes in the gas fill, thus diagnosing a variety of targets allows us to test the timedependence of the models.

\section{MEASUREMENT OF $\mathbf{T}_{\mathrm{e}}$}

Two types of target geometries are used in this study: bags of gas, which may be treated with a virtually time-independent analysis, and hohlraums, which require time-dependent analysis. The gasbags are formed by inflating $\mathrm{CH}$ membranes which are glued to both sides of a $2.75 \mathrm{~mm}$ diameter washer. The gas-filled hohlraums are Au cylinders $2.5 \mathrm{~mm}$ long and $1.6 \mathrm{~mm}$ in diameter. Thin $\mathrm{CH}$ membranes are placed over the endcaps of the cylinder to prevent the gas from escaping. Figure 1 shows a schematic of the two target geometries.

SIDE VIEW OF GAS BAG

SIDE VIEW OF HOHLRAUM

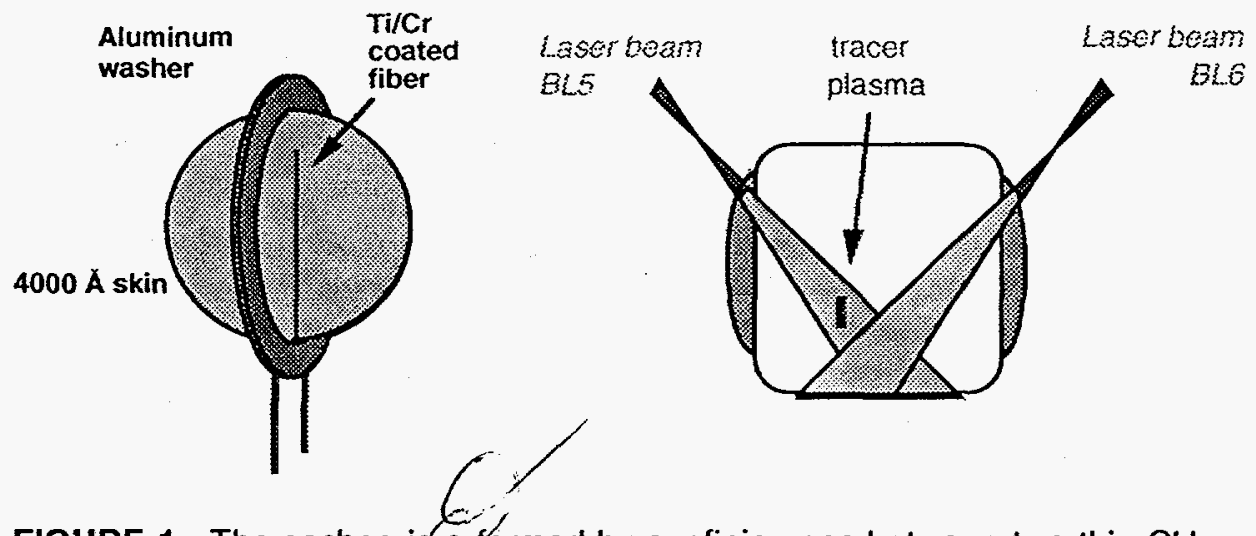

FIGURE 1. The gasbag is a formed by confining gas between two thin $\mathrm{CH}$ membranes. The plasma is formed by overlapping five laser beams on each membrane. The gas-filled hohlraum is an Au cylinder in which the laser beams enter through the endcaps which are covered by a thin $\mathrm{CH}$ membrane to enclose the gas.

The plasmas are produced by laser-irradiating the targets with approximately 20 $\mathrm{kJ}$ of laser energy. The laser ionizes the gas-filled target to form a plasma with an electron density, $\mathrm{n}_{\mathrm{e}}$, determined by the gas fill. For instance, neopentane, $\mathrm{C}_{5} \mathrm{H}_{12}$, filled to a pressure of 1 atm provides a density of $10^{21} \mathrm{~cm}^{-3}$ when it is completely ionized. Time-resolved x-ray diagnostics are used to monitor the plasma's volume, position, and spectra. These diagnostics provide a check of the density in the target because the X-ray images enable a measure of the expansion of the spectral emitter.

$X$-ray spectra used to diagnose the target are emitted from mid- $Z$ dopants. These are deposited on a thin $\mathrm{CH}$ substrate that is inserted into the target at a chosen position. The dopant coatings are typically only $2000 \AA$ to insure that their 
emission for the wavelength of interest is optically thin. To diagnose $T_{e}$, we use the ratio of the He-like lines from two different elements or the He-like lines and their Li-like satellites. An example of the x-ray spectrum of a mixed $\mathrm{Ti}$ and $\mathrm{Cr}$ dopant inside these targets is shown in Fig 2. The spectral lines identified in the figure are the two He-like $n=3-1$ transitions that are used in forming an isoelectronic line intensity ratio.

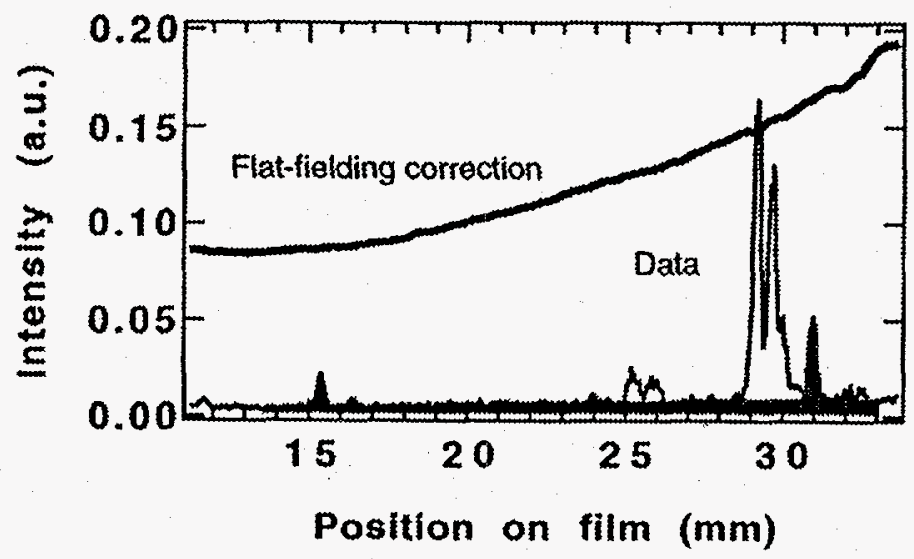

FIGURE 2. X-ray spectra emitted by the plasma of a typical dopant located inside a gas-filled target. The flat-fielding of the spectrometer is an important correction to the spectra and is measured for each instrument.

Because the temporal dependence is important, the spectra are recorded on gated microchannel plates which provide resolution in space and time (4). These instruments depend on fast electronics and this introduces two considerations for the measurements. First, the dynamic range of the instrument is approximately 40. This must be taken into consideration for choosing the optimal spectroscopic diagnostic. Second, the instrument detector response changes over the length of the data recording strip (5). A typical instrument can decrease by a factor of 2 in gain. For this reason, calibration of the instrument is performed for each microchannel plate and the response is unfolded from the measurement. An example of the detector response is shown in Figure 2.

Analysis of the spectrum relies on a collisional-radiative model of the plasma. In these examples we use the code, FLY, which sets up the rate matrix and solves for populations of K-shell ions (6). To look at the details more in-depth, we will consider a hypothetical test case to illustrate the behavior of the spectroscopic diagnostics, then we will show the results from three different targets. Depending on the target, differences of up to $30 \%$ are observed between the steady-state and non steady-state analysis.

\section{MODEL Te SLOPE}

For the test case, we consider a plasma that has a constant rise in $T_{e}$ and a constant electron density which is a simplified representation offaplasma conditions

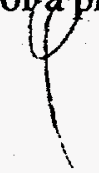


expected from the targets we will discuss later. Figure 3 shows a temperature slope that rises from 500 to $3500 \mathrm{eV}$ in $600 \mathrm{ps}$. Shown on the left hand abssissa is the ratio predicted for possible spectroscopic diagnostics. In the figure a commonly used ratio, the He-like $n=2-1$ resonance line to its Li-like satellites, He-like $\alpha / \mathrm{jkl}$, shoots up from 0 to 45 for Ar. Here, the plasma is hot enough to quickly strip through the Li-like ionization stage. This results in a very large ratio that requires a dynamic range that can surpass that of gated microchannel plate detectors for the highest temperatures. Another typical ratio, the Ly $\alpha$ to the He $\alpha$, has a significant temporal lag during the time frame of interest because of the temporal ionization lag to the hydrogen-like stage. The third ratio shown is the isoelectronic line ratio of $\mathrm{Cr}$ He-like $n=3-1$ to the Ti He-like $n=3-1$ transitions. It follows the input $\mathrm{T}_{\mathrm{e}}$ time history rise very well and is well within the sensitivity of the diagnostic. This case illustrates some of the advantages of using an isoelectronic line ratio which is discussed in other papers ( 7 ).

The test case was also calculated for different densities. The shaded area in figure 3 show the limits for densities of $2 \times 10^{21}-5 \times 10^{20} \mathrm{~cm}^{-3}$ for the isoelectronic ratio only. For the laser-produced plasmas in this study, the range of densities expected varies by a factor of 2 . Over this range, the ratio changes by a value of $10 \%$ which translates to a change of over $15 \%$ for the $T_{e}$ itself. This relatively small change enables us to investigate the temporal dependence of the line ratios without introducing significant errors due to possible density changes.

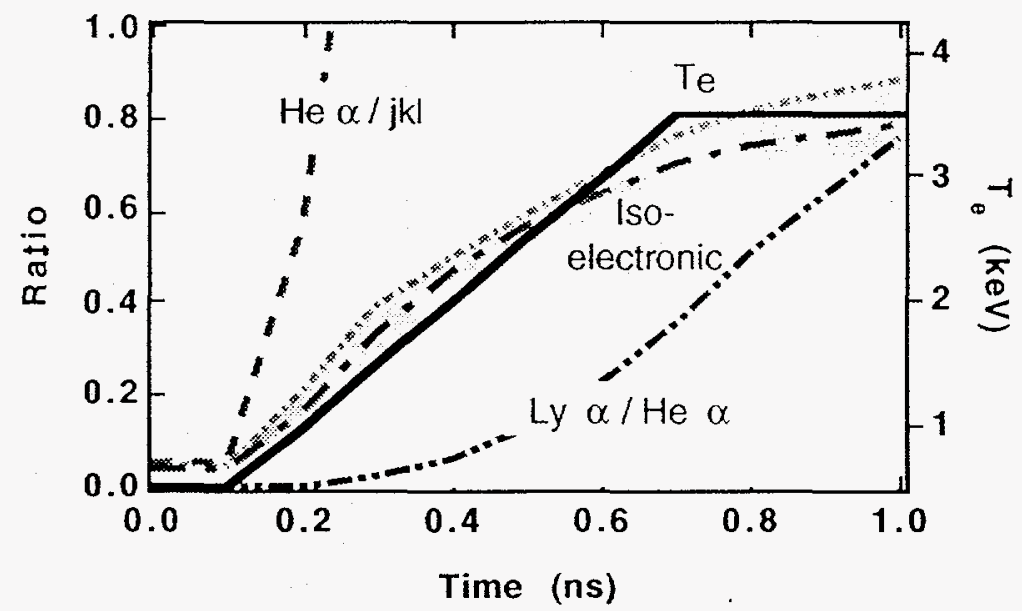

Figure 3. Three possible ratios that can be used to diagnose $T_{e}$. The isoelectronic ratio tracks the $T_{e}$ slope quite well. The shaded area correpsonds to a factor of 2 variation of density for the isoelectronic ratio. The $\mathrm{He} \alpha / \mathrm{jk}$ and the Ly $\alpha / \mathrm{He} \alpha$ are not ideal because they do not follow the $\mathrm{T}_{e}$ slope very well.

\section{GASBAGS}

To demonstrate the increasing time-dependence of plasmas, we begin with the gasbags. The gasbag targets are similar to the model plasma because the $T_{e}$ is 
similar and the density is virtually constant. After the initial ablation of the $\mathrm{CH}$ membrane, the gasbags form a quiescient plasma $\sim 2.5 \mathrm{~mm}$ in diameter $(8)$. According to computer simulations, the temperature rises very quickly during the 1 ns square heating laser pulse to achieve a peak temperature of $3-4 \mathrm{keV}$. X-ray images of the plasma volume have confirmed that the dopant in the neopentanefilled gasbags expands and equilibrates to a volume consistent with a density of $10^{21} \mathrm{~cm}^{-3}$ and remains stable during the second half of the $1 \mathrm{~ns}$ laser pulse. The disassembly of the gasbag plasma is due to the expansion of the plasma, therefore in the central region where the temperature measurements are performed, it is reasonable to assume a constant density during this time.

In this case, an iterative process is used to determine the $\mathrm{T}_{\mathrm{e}}$. First steady state values are determined from the measured ratios and are used to create the inital time history. Then a time-dependent run is performed to calculate ratios. These ratios are compared with the measured ratios and, based on these results, a corrected $\mathrm{T}_{\mathrm{e}}$ time history is produced. This processes is continued until it converges to a timedependent history that is consistent with the measured ratios. Since the plasma density remains constant in this case, the process converges in only 5 or 6 iterations.

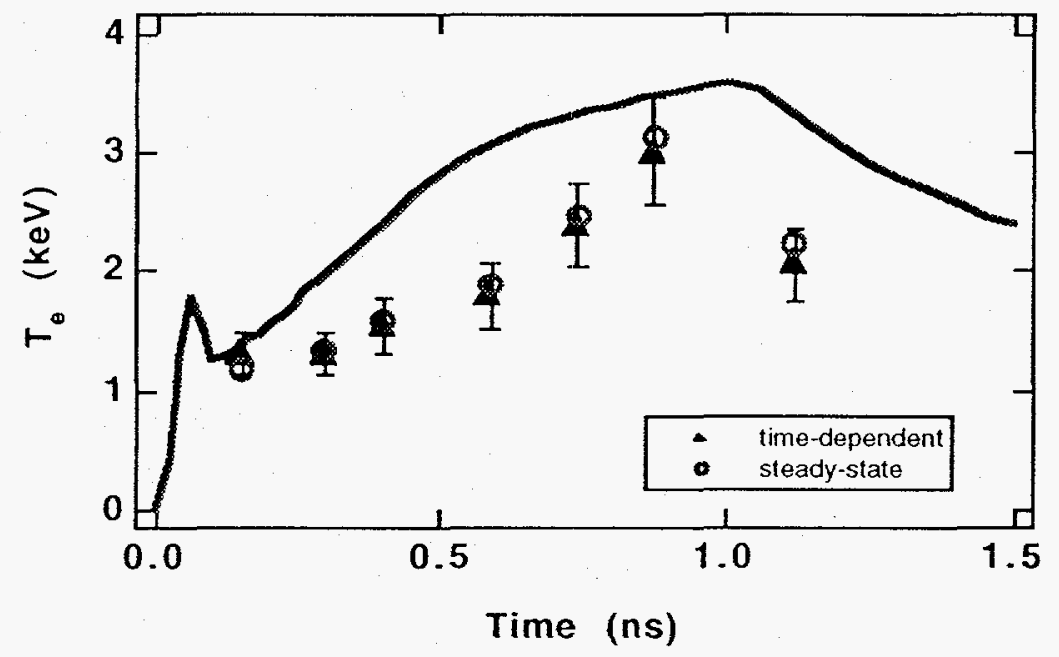

FIGURE 4. Graph of $T_{e}$ vs time for gasbags targets. The plasmas are homogeneous after $300 \mathrm{ps}$ and the volume at the center being measured is at constant density.

Figure 4 shows the Lasnex calculated temperature as well as the temperature deduced from steady state and time-dependent analysis. Temporal error bars for all measurements are $\pm 0.15 \mathrm{~ns}$. As there were a large number of shots, each point represents an average over many shots. There is only a $4 \%$ different from the peak temperatures in this case. The small difference between the time-independent and time-dependent analysis is not surprising since the density does not significantly change, and the electron density remains fairly high, $10^{21} \mathrm{~cm}^{-3}$. Although the peak temperature is consistent with the calculations, the rising temperature slope is inverted when compared to the calculations, a discrepancy which is still under investigation. 


\section{HOHLRAUMS}

Gas-filled hohlraums are more complex than gas bags because the electron density can change over the time of interest. Unlike gasbags, the hohlraum wall is thick and confines the plasma inside. Therefore, in addition, the laser energy that is not absorbed by the gas laser energy irradiates the wall of the hohlraum and produces an expanding Au plasma. Large hohlraums are $2.5 \mathrm{~mm}$ long by $2.5 \mathrm{~mm}$ in diameter and have roughly the same gas volume as the gasbags. The temperature history is similar and the density history is no longer constant, but increases towards the end of the $1.6 \mathrm{~ns}$ pulse (9).

Lasnex simulations of these targets predict a change in the density history of a factor of 2. For this reason, a simple fit to the ratios is no longer sufficient to deduce the temperatures. In these cases, it is more appropriate to initialize the timedependent analysis with the time-dependent hydrodynamic temperature and density histories. The data and plasma model results can be iterated to convergence by varying the $T_{e}$ as in the gasbag case. As discussed in the test $T_{e}$ slope case, the spectroscopic diagnostic is more sensitive to $T_{e}$ than to $n_{e}$. Hence, in this process, we assume the $n_{e}$ history does not vary, however, after the convergence on $T_{e}$, variations of the $n_{\mathrm{e}}$ are performed to verify this assumption.

For hohlraums we use isoelectronic line ratio measurements. This technique is well-suited to diagnose harsh plasma environments because it is better able to track the temporal history of ionizing plasmas. The ratio is temperature sensitive because of its exponential dependence on $1 / T_{e}$. In these measurements, we use the $n=3-1$ transitions because they are not optically thick and do not have underlying satellites for our plasma conditions, therefore they are reliable diagnostics.

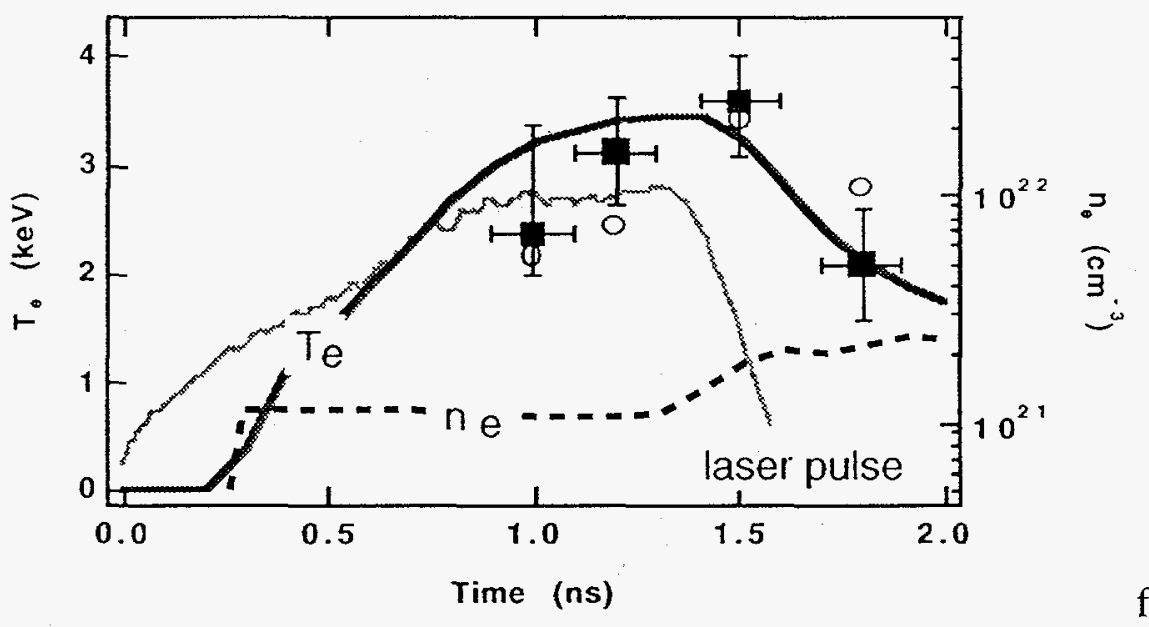

FIGURE 5. $T_{e}$ versus time for a large hohlraum. The circles denote the steady state values and the squares denote the time-dependent values. The calculated temperature history is similar to the gasbag history, but the density increases as the plasma is confined. 
Figure 5 shows the $T_{e}$ vs time for the large hohlraums. The ratios of the spectral lines are only measured after $1 \mathrm{~ns}$ to allow for sufficient ablation and equilibration of the tracer with the plasma formed by the gas. As in Fig. 4, we show the time-dependent and time-independent $T_{e}$ along with the Lasnex calculation. Since these targets were designed to be large scale-length plasmas, the $\mathrm{T}_{\mathrm{e}}$ history is similar to that of the gasbags. However, the steady-state $\mathrm{T}_{\mathrm{e}}$ is now in error by up to approximately $9 \%$ because both the density and temperature change as a function of time. By this method, we have measured the $\mathrm{T}_{\mathrm{e}}$ of large scale hohlraums to $\pm 15 \%$ of the calculated Lasnex value.

The smaller hohlraums are often referred to as Scale-1 hohlraums because they are the standard-sized targets used to implode a spherical capsule for laser fusion studies on Nova. These plasmas are very time-dependent and can be subjected to very strong radiation fields. The use of isoelectronic $\mathrm{Ti} / \mathrm{Cr}$ ratios is advantageous because it is able to track changing ionization balances, and, it is insignificantly sensitive to high radiation fields (10).

The analysis of these targets is more time-dependent because the typical gas-fill in these targets is lower in $n_{e}$. Methane produces an initial density of $0.6 \times 10^{21}$ $\mathrm{cm}^{-3}$ in the hohlraum. Furthermore, the heating of the hohlraum depends more on electron conduction and radiative heating than the other targets because less surface area of the hohlraum is directly irradiated by the beams.

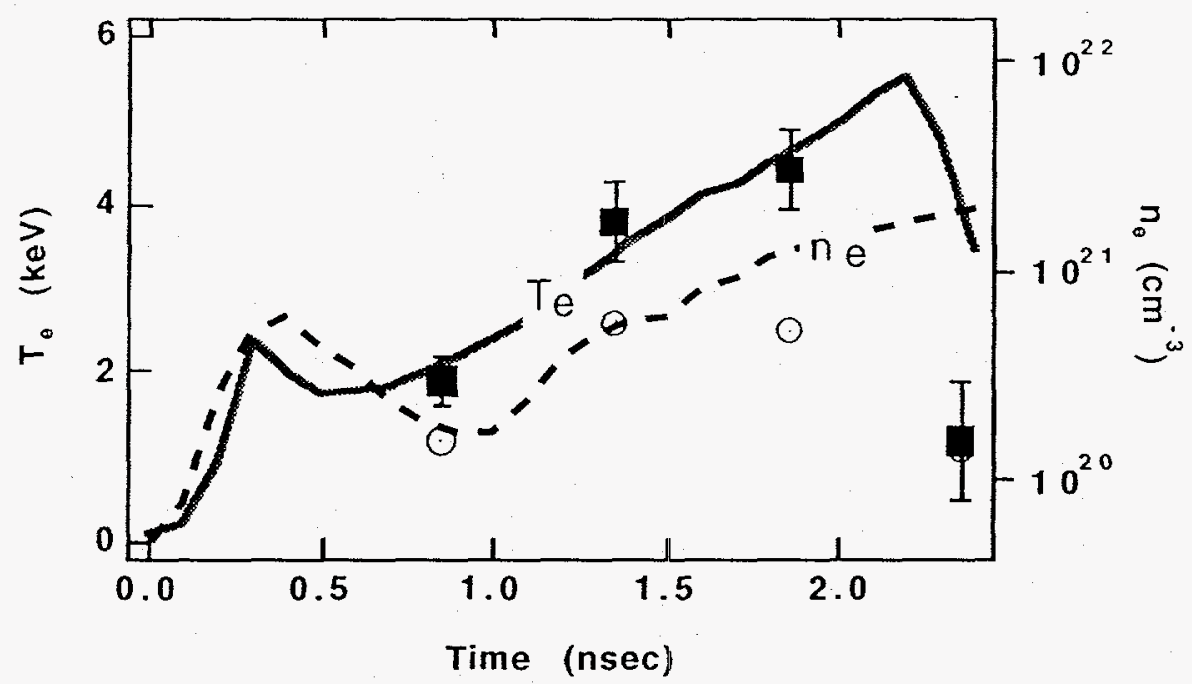

FIGURE 6. Measurements for scale-1 gas-filled hohiraums, $T_{e}$ vs time. The calculated $T_{e}$ history is monotonically increasing, however, the measured $T_{e}$ falls after $1.8 \mathrm{~ns}$. The circles denote the steady state values and the squares denote the time-dependent values.

For the analysis we show the temperature in a volume of the hohlraum directly irradiated by the lasers which is more analgous to the other two cases already presented. The data track the calculated history for $\sim 1.8 \mathrm{~ns}$, but then turn over and the temperature drops even though the hohlraum heating pulse is still on. Part of 
this discrepancy is due to scattering of laser light by the plasma (11). Measurements of scattering do increase during the second half of the pulse and the Lasnex temperature profile here has not yet been corrected for this. For these history, the $\mathrm{n}_{\mathrm{e}}$ changes by a factor of 10 . Images of the plasma dopant show that it does become more confined radially later in time which increases the density. The electron temperature due to a steady state calculation in this case would be in error by over $30 \%$ if no time-dependent analysis was performed.

\section{CONCLUSIONS}

Time-dependent measurements and analyses are important to correctly diagnose large laser-produced plasmas. Experiments in which a steady-state analysis is used to determine $\mathrm{T}_{\mathrm{e}}$ may introduce an error of up to $30 \%$. By a method of iteration, we find that we can measure the temperature of gasbags in the $2-3 \mathrm{keV} \mathrm{T}_{\mathrm{e}}$ range by standard methods. Gas-filled hohlraums require a more involved iteration process that uses a temperature and density history from calculations as the initial conditions. Electron temperature measurements of large gas-filled hohlraums have verified the hydrodynamic calculations which peak at $3.7 \mathrm{keV}$. The smaller scale-1 hohlraums are more challenging because the temporal histories vary more and current work shows that these hohlraums attain peak temperatures of $\mathrm{T}_{\mathrm{e}} \sim 4.5 \mathrm{eV}$, though the measurements do not agree with simulations at late times. These measurements allow us to assess the simulations and help us understand other related plasma processes occurring in the plasma.

\section{ACKNOWLEDGEMENTS}

We thank O. Landen and B. Hammel for useful discussions. This work was performed under the auspices of the U.S. Department of Energy by the Lawrence Livermore National Laboratory under Contract No. W-7405-ENG-48.

\section{REFERENCES}

1. Kauffman, R. L., "X-ray Radiation from Laser Plasma" in Handbook of Plasma Physics, vol. 3, eds. Rubenchik and Witkowski, 111-162 (Elsevier Science, North-Holland, 1991) and references therin.

2. J. C. Fernandez, et al Phys. Rev. E 53, 2747-2750 (1996).

3. Lindl, J. D., Phys. of Plasmas 2, 3933-4024 (1995).

4. Back, C. A. Kauffman, R. L., Bell, P. M., Kilkenny, J. D., Rev. Sci. Instrum. 66, 764-766 (1995).

5. Kilkenny, J. D., Laser and Part. Beams 9, 49-69 (1991).

6. Lee, R. W., Whitten, B. L. and Strout, R. E., J. Quant. Spectrosc. Radiat. Transfer 32, 91101 (1984); also see the FLY users manual available from R. W. Lee.

7. Marjoribanks, R. S., Richardson, M. C., Jaanimagi, P. A. and Epstein, R., Phys. Rev. A 46, R1747-R1750, (1992)

8. Kalantar, D. H., Klem, D. E., MacGowan, B. J., Moody, J. D., et al., Physics of Plasmas 2, 3161-3168 (1995).

9. Powers, L. V., Berger, R. L., Kauffman, R. L., MacGowan, B. J., et al, Physics of Plasmas 2, 2473-2479 (1995).

10. Shepard, T. D., Back, C. A., Kalantar, D. H., Kauffman, R. L., et al., Rev. of Sci.

Instruments 66, 749-751 (1995).

11. MacGowan, B. J., Physics of Plasmas, to be published May 1996. 